\title{
Enquête en « zone d'attente réservée » de l'aéroport de Paris-Charles de Gaulle : vers une gestion sécuritaire des « flux migratoires "
}

Social inquiry in the "restricted transit zone" of Paris-Charles de Gaulle airport:Towards the securitization of the "management of migration flows" Investigación en la "zona de espera reservada" del aeropuerto de París-Charles de Gaulle: hacia una gestión securitaria de los "flujos migratorios"

\section{Morgane Iserte}

\section{OpenEdition}

Journals

Édition électronique

URL : http://journals.openedition.org/conflits/15743

DOI : $10.4000 /$ conflits. 15743

ISSN : $1777-5345$

Éditeur :

CCLS - Centre d'études sur les conflits lilberté et sécurité, L'Harmattan

Édition imprimée

Date de publication : 30 octobre 2008

Pagination : $31-53$

ISBN : 978-2-296-06624-3

ISSN : 1157-996X

Référence électronique

Morgane Iserte, «Enquête en « zone d'attente réservée » de l'aéroport de Paris-Charles de Gaulle : vers une gestion sécuritaire des «flux migratoires » », Cultures \& Conflits [En ligne], 71 | automne 2008, mis en ligne le 05 février 2009, consulté le 30 mars 2021. URL : http://journals.openedition.org/conflits/ 15743 ; DOI : https://doi.org/10.4000/conflits. 15743 


\section{Enquête en "zone d'attente réservée " de l'aéroport de Paris-Charles de Gaulle : vers une gestion sécuritaire des «flux migratoires »}

\section{Morgane ISERTE}

Morgane Iserte est doctorante en sociologie au Centre d'études des mouvements sociaux, sous la direction de Louis Quéré. Elle est bénévole à l'Association nationale d'assistance aux frontières pour les étrangers (Anafé) depuis mars 2006.

T 'objet de cet article est de décrire certaines procédures du « dispositif 1 » Le contrôle migratoire relatives à l'entrée sur le territoire français et au transit en zone dite internationale des étrangers, telles que mises en œuvre dans la zone d'attente du site aéroportuaire de Paris-Charles de Gaulle (CDG). Nous souhaitons ici illustrer la mutation des techniques de confinement des étrangers au regard de l'évolution des exigences sécuritaires en matière de contrôle de l'immigration au sein de cette plate-forme aéroportuaire, qui constitue le principal lieu de passage d'étrangers non admis sur le territoire et d'arrivée de demandeurs d'asile en France. Chaque année, 11 millions de personnes sont contrôlés à la frontière de Roissy. Ce pôle est aussi une zone d'activité de premier plan où travaillent 90000 salariés dans 700 entreprises et dont la gestion représente un enjeu économique majeur. C'est enfin un « $h u b^{2}$ » international, c'est-à-dire un aéroport permettant

1. "Il est clair que le terme, dans l'usage commun comme dans celui qu'en propose Foucault, semble renvoyer à un ensemble de pratiques et de mécanismes (tout uniment discursifs et non discursifs, juridiques, techniques et militaires) qui ont pour objectif de faire face à une urgence pour obtenir un effet plus ou moins immédiat ", Agamben G., Qu'est-ce qu'un dispositif ?, Paris, Ed. Payot \& Rivages, 2007, pp. 20-21. Voir aussi : Peeters H., Charlier P., «Introduction. Contributions à une théorie du dispositif », Le Dispositif : entre usage et concept, Hermès, n²5, CNRS Editions, 1999: "A ce titre, le dispositif peut être défini comme la concrétisation d'une intention au travers de la mise en place d'environnements aménagés.", p. 18 ; "Le dispositif apparaît comme le concept par excellence de l'entre-deux. [...] L'entre-deux ne dissout pas les pôles, il les met en relation. Le dispositif désigne le lieu d'une dialectique qui demande à être traitée pour elle-même et qui doit être encore véritablement thématisée. ", pp. 22-23.

2. «Hub» (littéralement : moyeu) : il s'agit d'un concept développé dans les années 1980 par les 
aux passagers de changer rapidement et facilement de vol, où transitent chaque année près de 55 millions de passagers, véritable "point de connexion entre une multiplicité de flux ${ }^{3}$ ». Comme l'écrit Michel Serres : "Dans l'ensemble ruisselant $d u$ réseau, un tel noud joue le rôle d'un récepteur et d'un redistributeur, il synthétise et analyse, mélange, classe et trie, choisit et émet. Il importe et exporte ${ }^{4}$ ».

Notre analyse des techniques utilisées par les acteurs engagés dans «la lutte contre la pression migratoire aéroportuaire 5 », en premier lieu les agents de la Direction de la police aux frontières (DPAF) de Roissy/Le Bourget, s’intègre dans un cadre théorique global qui réfléchit, de façon pluridisciplinaire, aux questions posées par la mondialisation dans le domaine de l'étude des migrations internationales. Elle s'appuie sur un examen des pratiques frontalières à partir d'observations directes en zone d'attente et du recueil d'un matériau vaste, issu de sources officielles, académiques, journalistiques, commerciales et associatives ${ }^{6}$. Nous verrons ainsi que d'autres acteurs présents sur la plate-forme aéroportuaire de Paris-CDG sont aussi impliqués dans ce contrôle et nous aborderons la question des arrangements entre la police aux frontière (PAF) et ses deux principaux interlocuteurs à Roissy : Aéroports de Paris et Air France. Nous souhaitons ainsi souligner l'influence des logiques sécuritaires et managériales sur le travail policier de contrôle des frontières et les conséquences que celles-ci impliquent sur les techniques de confinement en zone d'attente. L'aéroport Paris-CDG peut, par bien des aspects, être considéré comme une "projection locale du réseau global 7 ", et notre propos est de saisir les conséquences du processus de rationalisation sécuritaire et de contrôle ${ }^{8}$ sur le dispositif de " gestion des flux migratoires » mis en œuvre à la frontière de Roissy.

Une zone d'attente, telle que définie par la loi du 6 juillet 1992, dite loi Quilès 9 :

compagnies aériennes américaines, destiné à optimiser le remplissage des vols. Son efficacité est renforcée par une certaine synchronisation des horaires des vols « moyens courriers » ( intérieurs » ou « internationaux ») et des vols « longs courriers », et par des atterrissages et décollages par vague. (Définition Wikipédia : http://fr.wikipedia.org/wiki/Hub).

3. Serres M., Hermès II, L'Interférence, Paris, Minuit, 1972, p. 130.

4. Ibid.

5. "Chapitre VI - L’immigration irrégulière », Les Orientations de la politique d'immigration, Secrétariat général du Comité interministériel de contrôle de l'immigration, Paris, La Documentation française, 2007, p. 119.

6. L'ensemble des notes de bas de page qui mentionnent «compte rendu de la visite de telle date » sont issues d'observations personnelles.

7. Serres M., op. cit., p. 132.

8. Deleuze G., « Post-scriptum sur les sociétés de contrôle », Pourparlers (1972-1990), Paris, Minuit, 1990 , pp. 240-247.

9. Voir la très complète présentation du régime de maintien aux frontières de François JulienLaferrière, «La rétention des étrangers aux frontières françaises ", Cultures E Conflits, $\mathrm{n}^{\circ} 23$, automne 1996, pp. 7-43. 
«s'étend des points d'embarquement et de débarquement à ceux où sont effectués les contrôles aux personnes. Elle peut inclure, sur l'emprise, ou à proximité de la gare, du port ou de l'aéroport ou à proximité du lieu de débarquement, un ou plusieurs lieux d'hébergement assurant aux étrangers concernés des prestations de type hôtelier 10 ».

Depuis la loi du 26 novembre 2003, dite loi Sarkozy :

« la zone d'attente s'étend, sans qu'il soit besoin de prendre une décision particulière, aux lieux dans lesquels l'étranger doit se rendre soit dans le cadre de la procédure en cours, soit en cas de nécessité médicale $11 »$.

En conséquence, la zone d'attente de l'aéroport de Paris-Charles de Gaulle comprend la zone d'attente pour personnes en instance (ZAPI 3, lieu d'hébergement " aux conditions hôtelières » de 164 places mis en service en 2001) et plusieurs hôtels de la zone aéroportuaire pour les mineurs isolés de moins de 13 ans à Roissy, l'hôpital Ballanger pour les malades à Aulnaysous-Bois, le Tribunal de grande instance de Bobigny, les tribunaux administratifs de Paris et de Cergy, la Cour d'appel de Paris, etc. : c'est-à-dire partout où se trouve une personne maintenue. Cette zone s'étend également sur l'ensemble des installations aéroportuaires, depuis le tarmac jusqu'aux locaux de la police où s'effectuent les contrôles des voyageurs. La zone d'attente recoupe ainsi la zone réservée - ou zone de sûreté à accès réglementé - de l'aéroport. C'est à cet espace, là où zone d'attente et zone réservée coïncident - la «zone d'attente réservée »-que nous nous intéresserons ici. Il s'agit principalement des locaux et salles de maintien dans les postes de police, situées dans chacun des 8 aérogares des 3 terminaux de Paris-CDG ${ }^{12}$.

L'accès à la zone d'attente est soumis à l'octroi d'une habilitation par le directeur des libertés publiques et des affaires juridiques, prise après avis $\mathrm{du}$ directeur central de la police aux frontières. Seuls les membres des associations habilitées par décret ${ }^{13}$ disposant d'un droit d'accès à la zone d'attente et titulai-

10. Article L. 221-2 du Code de l'entrée et du séjour des étrangers et du droit d'asile (CESEDA).

11. Complément à l'article cité.

12. Sauf dans l'aérogare D du terminal 2 (vols Shengen). Pour une description précise des lieux de maintien en aérogares, voir le rapport de l'Association nationale d'assistance aux frontières pour les étrangers (Anafé), Une France inaccessible, décembre 2007 (téléchargeable sur le site de l'Anafé : http://www.anafe.org), établi à partir d'observations réalisées lors de visites en aérogares à Roissy en 2006 et 2007.

13. Selon le décret du 30 mai 2006 (NOR : INTD0600504A), sont habilitées les associations suivantes qui font partie de l'Anafé : Accueil aux médecins et personnels de santé réfugiés en France, Amnesty International - section française, Anafé, CIMADE, France Terre d'asile, Forum réfugiés, Groupe accueil et solidarité, GISTI, Ligue des droits de l'Homme, Mouvement contre le racisme et pour l'amitié entre les peuples. Trois associations non membres de l'Anafé sont également habilitées : Médecins sans frontières, Médecins du monde, la Croix-Rouge française. 
res d'une carte de visiteur personnelle, les parlementaires et les juges des libertés et de la détention ${ }^{14}$ peuvent accéder à la zone d'attente. Quant à la zone réservée de l'aéroport, elle n'est pas non plus libre d'accès. Il faut, pour y circuler, être en possession d'un badge devant être visible à tout moment; les règles en matière de sûreté aéroportuaire sont drastiques. Nous avons pu accéder à la zone d'attente pour personnes en instance et dans les postes de police des aérogares de ParisCDG en zone réservée en tant que bénévole de l'Association nationale d'assistance aux frontières pour les étrangers ${ }^{15}$ (Anafé). La visite des terminaux s'effectue en compagnie d'officiers de la PAF après remise d'un badge vert de visiteur, ils nous accompagnent tout le long du « parcours », prévenant systématiquement leurs collègues de notre positionnement par radio. Il est formellement interdit, dans le cadre de la convention passée entre le ministère de l'Intérieur et l'Anafé, de nous entretenir avec des personnes « en cours de procédure » 16 . Ces restrictions ne sont pas sans poser de sérieuses limitations au travail d'enquête.

\section{L'évolution de la «pression migratoire aux frontières "}

L'instauration d'une police de l'immigration par une circulaire du 23 août $2005^{17}$ a pour objectif de renforcer la lutte contre l'immigration dite irrégulière, à la fois dans le domaine du contrôle aux frontières (entrée) et dans celui de l'éloignement (séjour) ${ }^{18}$. Le pilotage et l'animation de cette police ont été confiés à la direction centrale de la police aux frontières. La réalisation du premier volet de cette mission dans le cadre de la zone d'attente de la plateforme aéroportuaire de Paris-CDG est assurée par la direction de la police aux frontières (DPAF) de Roissy-Le Bourget, chargée du contrôle aux frontières et de la lutte contre les filières d'immigration illégale. L’objectif de la DPAF est de réduire les entrées irrégulières sur le territoire, ou encore la «pression migratoire exercée aux frontières », selon les termes d'un rapport du Comité interministériel de contrôle de l'immigration, le CICI ${ }^{19}$. Trois indicateurs

14. Ou les membres de la Commission nationale de contrôle des centres et locaux de rétention administrative et des zones d'attente, de la Commission nationale de déontologie de la sécurité, du Comité de prévention de la torture du Conseil de l'Europe ou du Haut Commissariat des Nations unies pour les réfugiés, selon des modalités et une périodicité déterminées d'un commun accord avec le ministre de l'Intérieur.

15. L'Anafé a été créée en 1989 afin de fournir une aide à caractère juridique et humanitaire aux étrangers en difficulté aux frontières françaises ; elle regroupe de nombreuses organisations de défense des droits des étrangers, des syndicats, des associations intervenant dans le champ de l'asile. Voir note $\mathrm{n}^{\circ} 13$.

16. Sur les conditions d'observation en aérogares, voir les rapports de campagne d'observations de l'Anafé, le dernier en date étant: Une France inaccessible, op. cit.

17. Circulaire du 23 août 2005 relative à la mise en place de la police de l'immigration, NOR : INT/C/05/00082/C.

18. «Afin d'accroître sa capacité de pilotage de la politique d'immigration, le service des étrangers et de la circulation transfrontière a entrepris de refondre son organisation interne ", Rapport d'activité du ministère de l'Intérieur 2004, chap. " Mission administration générale et territoriale de l'Etat », p. 18, disponible sur le site www.interieur.gouv.fr

19. «Chapitre VI - L’immigration irrégulière », op. cit., pp. 111-144. 
sont retenus dans ce document pour évaluer l'importance de cette "pression ». Il est intéressant ici de les rappeler, dans la mesure où ils définissent le cadre d'évaluation de la "pression migratoire aéroportuaire 20 » par la PAF. Nous voyons qu'ils entraînent un ajustement des procédures de contrôle de l'immigration aux frontières :

Le refoulement direct à la frontière (refus d'admission sur le territoire et réadmissions simplifiées). Cet indicateur "porte sur des personnes qui n'entreront pas sur le territoire de façon illégale, mais qui en ont eu la volonté ». Il concerne les étrangers qui se voient interdire l'accès au territoire français soit dès leur présentation à la frontière, soit après un placement en ZAPI 3, auxquels s'ajoutent les personnes renvoyées sans délai par les services de police dans le cadre de la procédure des "réadmissions, communément appelées simplifiées». Cette procédure permet à la PAF de renvoyer un étranger sans qu'aucune formalité particulière ne soit établie par les autorités frontalières au moment de son interpellation ${ }^{21}$.

Le placement en zone d'attente. Le nombre de placements en zone d'attente "peut donner une indication sur la pression migratoire, bien qu'il désigne des individus qui, pour la majorité d'entre eux, n'entreront pas en France ». Les personnes maintenues se répartissent en trois catégories : les demandeurs d'asile, qui demandent l'admission « au titre de l'asile » et passent en ZAPI 3 un entretien avec la division « asile » à la frontière de l'Office français de protection des réfugiés et apatrides (OFPRA) visant à établir que leur demande n'est pas «manifestement infondée 22 »; les non-admis et les personnes en transit interrompu, dont le refoulement ne peut être réalisé dans un délai de moins de quatre heures.

La demande d'asile à la frontière. Il est instructif de voir que, selon le CICI, "les demandeurs d'asile à la frontière constituent le troisième indicateur de la pression migratoire aux frontières ».

La «pression migratoire aux frontières » s'exerce en France pour l'essentiel à Paris-CDG, où se concentre plus de la moitié des tentatives d'entrée sur le territoire. A lui seul, cet aéroport a enregistré en 2005 plus de la moitié des refus d'admission prononcés (12000 non-admissions prononcées en $2005^{23}$, sur un

20. Ibid., p. 119.

21 . Elle se distingue de la réadmission exécutée en application d'une décision préfectorale (qui est considérée comme un éloignement, l'autre participant du contrôle des frontières).

22. Article L 221-1 du CESEDA : «L'étranger qui arrive en France [...] peut être maintenu dans une zone d'attente [...] pendant le temps strictement nécessaire à son départ et, s'il est demandeur d'asile, à un examen tendant à déterminer si sa demande n'est pas manifestement infondée. "

23. Audition devant la Commission des finances du Sénat en juin 2006, M. Jean-Yves Topin, contrôleur général de la PAF de Roissy, consultable sur le site : http://www.senat.fr/rap/r06045/r06-045_mono.html\#toc139 
total de 24654 en France et outre-mer) et près de $90 \%$ des placements en zone d'attente : 89,44 \% des 16157 placements en zone d'attente effectués en 2005 ont eu lieu à Roissy, alors qu'en 2006 ce chiffre s'élevait à 87,98 \% (14 427 personnes maintenues à Roissy sur 16397 placements). En outre, 91 \% des demandes d'admission au titre de l'asile sont formulées à Roissy.

L'évolution des indicateurs de la «pression migratoire aux frontières » nous apprend plusieurs choses. D’une part, elle est de mieux en mieux « mâेtrisée » : "c'est que les frontières sont bien gardées ! 24 ». En 2006, dans l'ensemble des zones d'attente de métropole et d'outre-mer, le nombre de personnes non admises sur le sol français est en diminution, passant de 24654 en 2005 à 22250 en 2006. Le nombre de placements en zone d'attente a lui aussi baissé : 16736 en 2005, contre 16397 en 2006. Quant au nombre de demandeurs d'asile à la frontière, il a beaucoup chuté depuis 2001, tout comme le taux d'admission de ces demandeurs : en 2006, 2727 personnes ont demandé l'asile à la frontière, $20 \%$ ont été admises au titre de l'asile, $66 \%$ pour toutes raisons confondues. En 2001, ces chiffres étaient de 10364 demandes : 17,2 \% admis au titre de l'asile, $94 \%$ toutes raisons confondues. Toutefois, pour être précis, le nombre de demandes d'admission au titre de l'asile a augmenté entre 2005 et 2006, tout particulièrement à Roissy où il est passé de 2424 à 2727 (soit près de plus de $20 \%$ ).

\begin{tabular}{|c|c|c|c|}
\hline $\begin{array}{l}\text { TABLEAU 1 : } \\
\text { Demandes d'asile } \\
\text { à la frontière 25 }\end{array}$ & $\begin{array}{l}\text { Nombre de } \\
\text { demandes }\end{array}$ & $\begin{array}{l}\text { Admissions au } \\
\text { titre de l'asile 26 }\end{array}$ & $\begin{array}{l}\text { Admissions toutes } \\
\text { raisons confondues }\end{array}$ \\
\hline 2001 & 10364 & $17,2 \%$ & $94 \%$ \\
\hline 2002 & 5912 & $15,2 \%$ & $75,2 \%$ \\
\hline 2003 & 2518 & $3,8 \%$ & $68,8 \%$ \\
\hline 2004 & 2424 & $7,8 \%$ & $48,9 \%$ \\
\hline 2005 & 2727 & $22,3 \%$ & NC \\
\hline 2006 & $20 \%$ & $66 \%$ \\
\hline
\end{tabular}

24. Compte rendu du 10 mai 2007, au T2C : le brigadier-chef K. nous accompagne lors de la visite des postes dans les aérogares du terminal 2.

25 . Source : ministère de l'Intérieur et de l'Aménagement du territoire (DLPAJ et DCPAF), "Statistiques relatives aux étrangers à la frontière" (juin 2007), consultable sur: http://www.anafe.org/stats.php, p. 1.

26. Ce pourcentage correspond aux avis favorables de l'OFPRA qui sont ensuite transmis au ministère de l'Intérieur, seule autorité prenant la décision finale. 
En outre, la durée de maintien en zone d'attente n'a cessé de diminuer, ce qui suggère une certaine accélération du rythme. La durée moyenne de maintien en zone d'attente en 2006 est de 1,89 jour alors qu'elle était de 5 jours en $2004{ }^{27}$. Le pourcentage de personnes maintenues après 48 heures a chuté de plus de $50 \%$ entre 2003 et 2005.

\begin{tabular}{|l|c|c|c|}
\hline $\begin{array}{l}\text { TABLEAU 2 : Maintenus } \\
\text { en ZAPI 3 et durée du } \\
\text { maintien } 28\end{array}$ & 2003 & 2004 & 2005 \\
\hline $\begin{array}{l}\text { Nombre de personnes } \\
\text { maintenus en zone d'at- } \\
\text { tente }\end{array}$ & 15498 & 15452 & 14451 \\
\hline $\begin{array}{l}\text { Personnes maintenues } \\
\text { après } 48 \text { heures }\end{array}$ & $50 \%$ & $23,91 \%$ & $21,26 \%$ \\
\hline $\begin{array}{l}\text { Personnes maintenues } \\
\text { après } 96 \text { heures }\end{array}$ & $43,6 \%$ & $16,19 \%$ & $14,45 \%$ \\
\hline $\begin{array}{l}\text { Personnes maintenues } \\
\text { après } 12 \text { jours }\end{array}$ & $19,25 \%$ & $2,89 \%$ & $2,55 \%$ \\
\hline
\end{tabular}

En 2006, le ministère de l'Intérieur a instruit $86 \%$ des demandes d'asile dans un délai inférieur à 4 jours ${ }^{29}$. Le taux d'occupation de la ZAPI 3 ne donc cesse de baisser. Il convient maintenant de nous pencher sur les facteurs qui permettent d'expliquer ces évolutions.

\section{Contrôle transfrontière et paradigme sécuritaire}

L'évolution à la baisse des indicateurs mesurant la «pression migratoire aux frontières » s'explique en premier lieu par la réorganisation du travail des services de la PAF et l'augmentation de ses effectifs à Roissy (augmentation de $25 \%$ en 5 ans, passant de 1356 fonctionnaires au 1 er janvier 2002 à 1689 fonctionnaires au 1 er janvier 2006). Elle est également le résultat d'une politique de l'immigration qui multiplie les «barrières à l'entrée ", tant au niveau national qu'aux niveaux européen et international : nouvelle procédure de délivrance des visas par les autorités consulaires, coopération accrue des transporteurs, collaborations renforcées entre les différentes polices et multi-

27. Statistiques Anafé, juin 2007.

28. Source : MIAT (DCPAF), Tableau $n^{\circ} \mathrm{VI}-8$, Les Orientations de la politique d'immigration, op. cit., p. 119.

29. Nous rappelons ici que la procédure de maintien en zone d'attente est une procédure administrative, qui n'est validée par un juge des libertés et de la détention qu'au bout de 96 heures (4 jours) où elle devient alors une procédure judiciaire. 
plication des «mesures de dissuasion aéroportuaires 30 " (voir infra). Enfin, l'agencement d'une nouvelle architecture sécuritaire du contrôle à Roissy constitue un autre facteur.

\title{
"Faire de l'aéroport Paris-CDG un espace de sécurité »
}

Lors d'un déplacement à l'aéroport Paris-CDG, le 20 avril 2006, Nicolas Sarkozy, alors ministre de l'Intérieur et de l'Aménagement du territoire, déclare :

\begin{abstract}
«La menace terroriste nous impose en permanence d'adapter nos postures et nos dispositifs : [...] il s'agit pour nous d'être en permanence en mesure de détecter le plus tôt possible les individus dangereux. A une époque où les flux de voyageurs sont considérables, où la notion même de frontière a changé de nature, cette idée de ciblage des individus ou d'éléments anormaux, et de traçabilité (c'est-à-dire la capacité que nous nous donnons de reconstituer un parcours) est au cœur du débat. Ces deux principes d'action, ciblage et traçabilité, nous permettront de relever le défi de la sécurité dans les années à venir. »
\end{abstract}

Le défi de la sécurité est le leitmotiv des gouvernements occidentaux depuis bien des années, et les attentats du 11 septembre 2001 n'ont fait qu'accentuer cette tendance. Ces discours sécuritaires affirment que les transformations de la modernité et l'accélération de la globalisation sont génératrices d'incertitudes que les nouvelles technologies sont appelées à maîtriser et à gérer. La «menace » est agglomérée dans un « continuum de sécurité »: il faut contrôler de la même façon l'immigration, la criminalité et le terrorisme, puisque ces phénomènes - et leur contrôle - sont associés ${ }^{31}$. Le développement des technologies de sécurité contribue à la mise en place d'un système global de surveillance "dont l'objet focal est l'individu, et l'outil stratégique toute information se rapportant à lui 32 » et engendre la mise en pratique de nouvelles mesures de «sûreté aéroportuaire ».

L'accès à la zone réservée de Paris-CDG est strictement réglementé : tous les accès entre zone publique et zone réservée (délimitée sur toute sa périphérie par une clôture hermétique) sont verrouillés ou contrôlés aux postes d'inspection filtrage (PIF) où sont effectuées plusieurs opérations - fouilles, moyens de détection (portique détecteur, rayons X), palpations de sécurité -

30. Les Orientations de la politique d'immigration, op. cit., p. 115.

31. Bigo D., Polices en réseaux, l'expérience européenne, Paris, Presses de la Fondation nationale des sciences politiques, 1996.

32 . Ceyhan A., «Technologie et sécurité : une gouvernance libérale dans un contexte d'incertitudes », Cultures $\mathcal{E}$ Conflits, nº4, 2006, p. 24. 
en vue de déceler des articles prohibés. Ne peuvent accéder en zone réservée que les personnes munies du badge ad hoc, indiquant les secteurs ${ }^{33}$ auxquels il donne accès. Depuis juillet 2004, ces badges comportent des données biométriques : le personnel des aéroports parisiens dispose de badges auxquels a été ajoutée une puce. A chaque passage par un poste d'inspection et filtrage pour accéder en zone réservée, le badge est lu, identifié par les données biométriques, et la photographie de son titulaire s'affiche sur un écran. Le surveillant vérifie que l'image qui apparaît est bien celle de la personne qui se trouve devant lui et contrôle sa pièce d'identité. L'adoption de la biométrie "fluidifie le passage des personnels 34 » en même temps qu'elle apporte un "véritable renforcement de la sécurité 35 ». L'attribution des badges d'accès suit une procédure très contraignante pour les personnes qui travaillent sur la plate-forme : ils sont octroyés par le Préfet de Seine-Saint-Denis, après consultation de la Direction des libertés publiques et des affaires juridiques (DLPAJ) et de la PAF ${ }^{36}$. Depuis la loi du 23 juillet $2006{ }^{37}$ sur la lutte contre le terrorisme, de nouvelles dispositions relatives à la sécurité et aux contrôles transfrontaliers ont été adoptées pour faciliter le recueil et l'exploitation des données relatives aux informations sur les voyageurs APIS / PNR (Advanced Passenger Information System / Passenger Name Record) ${ }^{38}$. La transmission des données, jusque-là « réactive " (les compagnies aériennes répondant aux demandes policières concernant les suspects spécifiques), devient largement «proactive ». Autre donnée concernant la sécurisation du site de Roissy : depuis 2003, Aéroports de Paris installe massivement des caméras de vidéo-surveillance dans l'aéroport. Le budget de ce projet est de l'ordre de 10 millions d'euros par an sur 5 ans, avec pour objectif de placer 6800 caméras. Des méthodes similaires sont appliquées aux terroristes, aux étrangers et aux employés : des catégories-cibles sont élaborées et soumises à

33. Voir Linhardt D., «L’économie du soupçon. Une contribution pragmatique à la sociologie de la menace ", Genèses, n44, septembre 2001, p. $82:$ : $A$ : proximité immédiate de l'avion en situation d'embarquement ou de débarquement des passagers, des bagages et du fret; $B$ : salles de tri, de conditionnement et de stockage des bagages ; F : fret ; $P$ : salles d'embarquement en service, et à l'arrivée, les zones de circulation des passagers jusqu'à l'entrée des salles de livraison bagages. »

34. Sur le site du Paris Air Show 2005, Toute l'actualité du groupe SAFRAN au 46e Salon du Bourget, «La sûreté aéroportuaire, au doigt et à l'œil », 18 juin 2005, http://www.parisairshow2005.com/fr/a_1806_3.php

35. Ibid.

36. C'est d'ailleurs l'une des raisons pour lesquelles, nous le verrons, les personnels travaillant sur la plate-forme de Roissy sont très prudents : la PAF peut demander au Préfet de leur retirer leur badge, précieux outil de travail, au moindre « écart » de leur part.

37. Loi n²006-64 du 23 janvier 2006 relative à la lutte contre le terrorisme et portant dispositions diverses relatives à la sécurité et aux contrôles frontaliers. NOR : INTX0500242L.

38 . De manière générale, les compagnies aériennes et les agences de voyage collectent ces informations auprès des passagers dans le cadre des services de réservation. Stockées dans les bases de données des systèmes de réservation, elles sont échangées entre les entreprises intervenantes à partir du moment de la réservation jusqu'à la réalisation des prestations demandées par les passagers. Les données présentes dans ces bases prennent la forme d'enregistrements d'informations standardisés au plan international dénommés «PNR ( Passenger Name Record). Pour plus d'informations sur le PNR, voir : http://www.cnil.fr/index.php?id=2101 
une surveillance accrue, afin de faciliter «la gestion de ces séries ouvertes, qui caractérise le mécanisme de sécurité 39 ».

Il convient de rappeler que Paris-CDG est :

« le bub d'Europe où l'on calcule le maximum d'interconnexions quotidiennes : 17000 pour Paris, 9000 pour Francfort, 5000 pour Heathrow ; de plus, les passagers en transit représentent $35 \%$ de l'ensemble des passagers. [...] Les grands bubs mondiaux, ces "nœuds" de correspondance, se livrent une concurrence sans merci pour attirer les voyageurs en transit. Ce qui convainc le voyageur en dernier lieu, c'est la praticabilité de l'aéroport, les services offerts, le " $h u b$ " dans lequel il a passé le temps le plus plaisant et le moins fatigant entre deux avions 40 ».

L'objectif des acteurs commerciaux de la plate-forme est donc de fluidifier les contrôles et d'éviter les engorgements, ce que doit prendre en compte la PAF tout en continuant «à assurer ses missions de sécurité publique ${ }^{41}$ ». Jean-Yves Topin, contrôleur général de la DPAF de Roissy en 2006, explique :

« la police est de plus en plus considérée par ADP et Air France comme un simple prestataire de services. La culture d'ADP s'apparente à celle d'un centre de profit et cet établissement tend à considérer l'Etat "comme un client comme les autres" ${ }^{42}$. "

Il indique par ailleurs que la DPAF compte sur certaines avancées technologiques pour "mener à bien ses missions et améliorer la qualité du service rendu »: la lecture optique des passeports (COVADIS ${ }^{43}$ ), le système de

39. Foucault M., Sécurité, territoire, population: Cours au Collège de France (1977-1978), Paris, Editions de l'Ecole des Hautes Etudes en sciences sociales, 2004, p. 22.

40. Pierre Graff, président d'Aéroports de Paris (ADP), lors d'un séminaire organisé le 10 mai 2005 à l'Assemblée nationale sur le thème des « aéroports au service du développement économique».

41. Audition de la Commission sur le fonctionnement administratif et budgétaire de la Direction de la PAF de Roissy/Le Bourget (7 juin 2006), Rapport d'information fait au nom de la commission des Finances, du contrôle budgétaire et des comptes économiques de la nation sur la réforme de l'Etat et les audits de modernisation, par M. Jean ARTHUIS, octobre 2006, http://www.senat.fr/rap/r06-045/r06-045_mono.html\#toc139

42. Ibid.

43. "COVADIS est un système de lecture optique automatisé des documents de voyage, avec interrogation automatique du fichier des personnes recherchées, dont le but est de fluidifier le passage aux postes frontières tout en renforçant la sécurité. Le temps de contrôle passe ainsi de 1 ou 2 minutes à environ 8 secondes. 2004 a permis la commande de 318 bornes de lecture. 108 ont été installées dans les ports ou aéroports de Bastia, Ajaccio, Marseille, Nice, Sète, Genève, Calais, Douvres, Dunkerque, Bordeaux, Toulouse, Strasbourg, Bâle-Mulhouse, Cherbourg, en complément des 191 déployées en 2003 à Roissy, Orly et pour l'Eurostar. Les autres le seront en 2005 dans les ports non encore équipés ", Rapport d'activité du ministère de l'Intérieur 2004, op. cit., p. 30. 
contrôle automatique des passagers européens (système Fast track, fondé sur un abonnement), ou encore le visa biométrique.

"Gérer les flux en vue d'un contrôle optimum »

Comment contrôler et catégoriser tous ces mouvements transnationaux de population?

- Grâce aux nouvelles technologies de surveillance :

La police de l'immigration a toujours eu recours à la technologie identitaire pour rationaliser le contrôle des étrangers ${ }^{44}$. Comme nous venons de le voir, l'intensification de la surveillance du mouvement se réalise à la fois par l'élargissement (en augmentant les échanges des données personnelles) et l'approfondissement (en introduisant la biométrie) des contrôles ${ }^{45}$. Ce processus se traduit par une forte discrimination entre "touristes » et «vagabonds ", pour reprendre la terminologie de Zygmunt Bauman ${ }^{46}$. Les moyens et les cibles des contrôles frontaliers sont en effet différenciés selon la nationalité ou la provenance des personnes.

S’agissant des contrôles pour entrer sur le territoire, le premier critère pratique établit une distinction entre étrangers « ressortissants de pays tiers » et membres de l’Union européenne (plus largement de l'Espace économique européen et de la Confédération helvétique), vérifié lors du contrôle des documents aux postes de contrôle frontalier par des agents de police. "Ce zonage est le fruit d'une réflexion qui trouve son origine dans le constat de la nécessité d'une meilleure maîtrise des flux à l'intérieur de l'aéroport ${ }^{47}$. " Il s'agit notamment de veiller à ce que les passagers qui sont contrôlés soient bien séparés de ceux qui ne le sont pas. Une illustration de cette « séparation » est l'installation en 2005, au terminal 2 F (qui fait partie du hub d'Air France, avec le terminal 2 E et son nouveau satellite d'embarquement $S 3$ ), de deux systèmes expérimentaux distincts d'identification biométrique, appelés BIODEV et PEGASE.

BIODEV est un dispositif de contrôle "ouvert à l'étranger 48 ». Il a été lancé le 22 mars 2005 sous forme expérimentale dans les consulats français de Bamako (Mali), de Colombo (Sri Lanka), de Minsk (Ukraine), de San

44. Voir notamment Noiriel G., Réfugiés et sans-papiers. La république face an droit d'asile $X I X^{e}-X X^{e}$ siècle, Paris, Calmann-Lévy, 1991 ; Spire A., Etrangers à la carte. L'administration de l'immigration en France (1945-1975), Paris, Grasset, 2005.

45 . Mitsilegas V., "Contrôle des étrangers, des passagers, des citoyens : surveillance et antiterrorisme », Cultures $\mathcal{E}$ Conflits, ${ }^{\circ} 58$, pp. 155-181.

46. Bauman Z., Le Cô̂t humain de la mondialisation, Paris, Hachette Littératures, 1999, chap. IV « Touristes et vagabonds ", pp. 119-155.

47 . Linhardt D., op. cit., p. 82.

48. Compte rendu de la visite du 21 juin 2006, au T2F : propos du major P.-A. qui nous accompagne. 
Francisco (Etats-Unis) et d'Annaba (Algérie). Il s'agit d'y établir des visas avec un composant électronique où figurent les empreintes digitales du détenteur de visa, et de les comparer au poste frontière avec celles du passager contrôlé. Ce système permet d'établir une traçabilité électronique de l'entrée et de la sortie des étrangers munis d'un visa biométrique. Un projet d'extension du champ géographique de l'expérience, BIODEV II, est à l'étude pour généraliser ce dispositif à d'autres "pays sources de l'immigration irrégulière vers la France 49 " (notamment au Maghreb et en Afrique subsaharienne). Il participe du projet conjoint de certains pays de l'Union pour mettre en place un système européen «VIS » d'information sur les visas de court séjour. Sa finalité est de «faciliter l'instruction et la délivrance des visas des demandeurs de bonne foi ainsi que les contrôles à l'entrée dans l'espace Schengen 50 ».

Un autre système d'identification biométrique est expérimenté depuis juin 2005 auprès de 10000 voyageurs réguliers d'Air France, tous volontaires. PEGASE, le Programme d'expérimentation d'une gestion automatisée et sécurisée, permet au passager affilié à ce programme de passer dans la zone sécurisée plus rapidement, sans contrôle, grâce à la lecture d'une empreinte digitale préalablement enregistrée qui actionne l'ouverture d'un sas. "Le voyageur y gagne du temps et la police y gagne une meilleure gestion des flux 51 ». La compagnie aussi... Selon Air France, qui a entièrement financé le projet (décidé conjointement avec le ministère de l'Intérieur et ADP) :

«PEGASE a permis de fluidifier le passage de la zone publique à la zone privée d'embarquement. La compétition ne se fait plus dans les airs, mais au sol. Le temps d'attente au niveau de la police est toujours un goulot d'étranglement qui se traduit par du stress et de l'inconfort pour nos clients ${ }^{52}$. »

Ce programme va être généralisé sous la forme du système PARAFES : Passage automatisé rapide aux frontières extérieures Schengen. Il est, comme PEGASE, réservé aux résidents de l'UE et de la Confédération helvétique. Le poste de police du terminal $2 \mathrm{E}$ possède un instrument encore plus sophistiqué (dont les coûts sont encore plus élevés) qui permet aussi de contrôler la

49. Rapport de commission d'enquête du Sénat $n^{\circ} 300$ : Immigration clandestine : une réalité inacceptable, une réponse ferme, juste et bumaine (2005-2006), tome 1, chap. 2 «Prévenir » I.A.3 «Accélérer la mise en commun des moyens » : http://www.senat.fr/rap/r05-300-1/r05-300-128.html\#toc181

50. Document « Coopération franco-allemande en matière de biométrie - BIODEV II », disponible sur le portail franco-allemand : http://www.france-allemagne.fr/Biometrie.html

51 . Intervention de N. Sarkozy à Roissy, 20 avril 2006 : http://www.interieur.gouv.fr/misill/sections/a_l_interieur/le_ministre/interventions/archives-sarkozy-2005-2007/2006-04-20roissy/view

52. Puel H., "Identification biométrique : un succès pour Air France ", 01 net, mercredi 6 juin 2007. 
température et le flux du sang du doigt pour pouvoir reconnaître un cas d'implantation artificiel du doigt.

L'avantage de ces systèmes différenciés de contrôle aux frontières réside dans le fait que "le contrôle automatique de certains passagers permet de mieux employer les policiers, de les recentrer sur les vols sensibles et d'accroître notre efficacité 53 ». La ségrégation des tâches de contrôle engendre un double résultat pour la PAF : le contrôle pour les touristes est fluidifié (en matière de contrôle aux frontières, le taux de satisfaction du public mesuré par ADP est de l'ordre de $90 \%$ !) et celui des « étrangers suspects » est renforcé.

\section{- En modifiant les modalités du transit aéroportuaire :}

Le contrôle aux frontières consiste aussi à surveiller les personnes qui transitent par la zone dite internationale de l'aéroport Paris-CDG. La question du transit aéroportuaire est centrale dans la gestion de la lutte contre l'immigration dite irrégulière En effet, jusqu'à présent, de nombreuses personnes profitaient d'un transit par Paris pour déposer, dans l'un des postes de police des aérogares de Roissy, leur demande d'admission au titre de l'asile. Depuis 1995, la France a mis en place, pour prévenir ces situations, un visa de transit aéroportuaire (VTA). Ce visa est requis pour certains voyageurs afin de pouvoir passer par la zone dite internationale d'un aéroport, sans accéder au territoire national du pays concerné, à l'occasion d'une escale ou d'un transfert entre deux tronçons d'un vol international ${ }^{54}$. Son obtention n'autorise en aucun cas l'entrée sur le territoire français. La France exige ce visa des ressortissants de 34 Etats 55 dont provient une majeure partie des demandeurs d'asile. D'ailleurs, l'instauration d'un VTA pour les ressortissants d'un pays se produit en général peu de temps après l'arrivée d'un grand nombre de personnes originaires de ce pays ayant cherché à demander l'asile en France à l'occasion d'un transit. Ce fut le cas le 7 avril 2003 pour la Côte d'Ivoire alors que des Ivoiriens fuyaient la crise qui avait éclaté en septembre 2002. Ce fut aussi le cas le 21 décembre 2006 lorsque l'instauration d'un VTA pour les ressortissants colombiens sanctionna l'«afflux massif » de Colombiens en ZAPI 3 à l'automne 2006. Ce fut encore le cas pour les Tchétchènes le 1 er février 2008. Sur les courbes recensant le nombre des personnes demandant l'admission au titre de l'asile, à chaque nouveau VTA, on voit très clairement que le nombre de demandeurs d'asile à la frontière chute ${ }^{56}$.

53 . Intervention de N. Sarkozy à Roissy.

54 . Cette exigence est une exception au privilège général de transit sans visa par la zone internationale de transit.

55. Pour obtenir la liste des pays dont les ressortissants sont soumis au VTA, consulter: www.anafe.org/download/texte/Liste\%20des\%20nationalit\%E9s\%20soumises $\% 20$ au $\% 20$ VTA.pdf (situation en février 2008).

56. Voir le graphique p. 6 dans le document « Statistiques relatives á la situation des étrangers aux frontières ", Anafé, 2007: http://www.anafe.org/download/generalites/stats-za-juin07.pdf 
Le visa de transit (ou visa d'escale) est délivré aux demandeurs qui souhaitent se rendre d'un Etat tiers (hors Schengen) à un autre Etat tiers (hors Schengen) en traversant la France, et ce pour une durée de 5 jours maximum. Il est bien sûr valable dans tout l'espace Schengen. Le refus de visa d'escale est une des procédures courantes de non-admission sur le territoire. Les critères de l'octroi et du refus de visa se fondent sur l'appréciation par l'officier de quart de l'absence de risques sérieux pour la sécurité publique ou de risque migratoire ; l'officier n'a pas à motiver sa décision. Lors d'un entretien avec un agent d'Air France en charge des correspondances au terminal $2 \mathrm{~B}$, ce dernier constate :

« il y a encore trois-quatre ans, il était possible de discuter avec l'officier de police judiciaire et nous obtenions facilement des visas d'escale pour nos passagers. Maintenant, ils sont plus sélectifs et il arrive que des personnes restent coincées en zone internationale en attendant leur vol. S’il y a un retard, elles peuvent y passer la nuit ${ }^{57}$. »

\section{- En interceptant les étrangers de "provenance inconnue»:}

L'une des missions de la police aux frontières consiste à minimiser le nombre des personnes qu'elle ne pourrait pas identifier ou dont elle ne pourrait déterminer la provenance : la capture des personnes «de provenance inconnue » est un objectif majeur de la PAF. En effet, elle ne peut en principe procéder au renvoi d'un étranger non admis que si elle connait son pays de provenance. Dans le cas contraire, il sera admis sur le territoire français, au plus tard à l'expiration de la durée maximale du maintien en zone d'attente qui est de 20 jours. En 2004, les personnes « de provenance inconnue » représentaient près de $30 \%$ de l'ensemble des maintenus. C'est bien pour cette raison que la PAF a multiplié et renforcé les moyens permettant d'identifier la provenance des étrangers, pour être sûre de pouvoir les refouler en cas de refus d'entrée.

L'une des premières modalités pratiques consiste à cibler un certain nombre de vols considérés comme présentant un « haut risque migratoire » et à y réaliser des contrôles en porte d'avion : ce sont les «contrôles passerelle». Une unité spéciale de la PAF - la Brigade mobile d'intervention (BMI) - est chargée de réaliser ces contrôles qui ont lieu directement à l'intérieur du couloir de débarquement. Les agents demandent aux personnes considérées comme suspectes de former un groupe séparé des autres voyageurs. Ces dernières sont ensuite accompagnées au poste de quart "pour vérifications ». Le « contrôle passerelle » se focalise sur le «maintien général » des personnes (il s'agit, pour les policiers, d'estimer quels étrangers semblent avoir le «niveau social adéquat » pour faire du tourisme...), le repérage des documents falsifiés et le parcours des voyageurs, rapidement soupçonnés lorsque leur voyage cor- 
respond à des itinéraires qui, selon la BMI, relèvent de filières de passeurs (par exemple, un vol Abidjan-Pékin via Paris). Ces contrôles ont lieu sur des destinations «sensibles » dont il existe une liste écrite à destination des agents de la PAF, mais aussi des membres des compagnies aériennes opérant à ParisCDG. Ces compagnies ont d'ailleurs pleinement intégré le principe des «contrôles passerelle» dans leur fonctionnement. Un major nous explique :

«Sur les gros vols, comme São Paolo ou Hong Kong, nous sommes en liaison avec la compagnie au départ qui nous prévient des incongruités. Comme, par exemple, des paysans ou des étudiants qui voyagent pour une semaine alors que le prix du billet est bien trop cher pour eux ${ }^{58}$. »

En 2005, les « contrôles passerelle » ont été systématisés sur les lignes les plus sensibles, à « haut risque migratoire ». Ainsi 14924 vols ont fait l'objet de contrôles, lesquels ont conduit à l'identification de 8154 étrangers en situation irrégulière. Chaque jour, la police effectue en moyenne 40 contrôles en porte d'avion dans l'ensemble des terminaux de Roissy ; les effectifs des agents effectuant ce type de contrôle sont passés de 18 à 60 en l'espace de deux ans.

Une autre mesure consiste à s'assurer de la traçabilité des étrangers, en contraignant les transporteurs à numériser les documents de voyage de ses passagers. L'article L625-1 du CESEDA instaure des amendes pouvant aller jusqu’à 5000 euros aux transporteurs qui débarquent sur le territoire français un étranger non ressortissant d'un Etat de l'Union européenne et non muni de document de voyage ou du visa qui lui est applicable. Les vérifications documentaires exigées peuvent être effectuées par un agent d'embarquement. En pratique, dans les aéroports ouverts au trafic international, les compagnies aériennes font appel à des sociétés de services dont les agents sont spécialement formés aux techniques de rapprochement documentaire. L'Etat contraint les transporteurs à jouer un rôle de "contrôleurs de frontières » et "délègue à des acteurs privés et transnationaux une partie de leur autorité, qui opèrent à des points situés en amont de l'entrée sur le territoire comme une série d'écluses, connues sous le nom de "contrôle à distance" 59 ». En 2003, 678 amendes ont été prononcées à l'encontre de compagnies aériennes; en 2004, ce chiffre est passé à 1033 60. Cette amende peut être ramenée à 3000 euros par passager "lorsque l'entreprise a mis en place et utilise, sur le lieu d'embarquement des passagers, un dispositif agréé de numérisation et de transmission, aux autorités françaises chargées $d u$ contrôle aux frontières, des

58. Compte rendu de la visite du 14 novembre 2006, au T2A.

59. Guiraudon V., « Logiques et pratiques de l'Etat délégateur : les compagnies de transport dans le contrôle migratoire à distance », Cultures \& Conflits, n45, 2002, pp. 51-63.

60. Chiffres fournis par la DLPAJ au titre des amendes aux transporteurs visées à l'article L625-1. 
documents de voyage et des visas 61 ». Depuis le 11 octobre 2006, ce dispositif de numérisation et de transmission des documents de voyage a été approuvé par décret ${ }^{62}$. L'entreprise de transport numérise à l'embarquement l'ensemble des documents de voyage présentés par chaque passager et stocke ces données sur un CD-Rom. Ce disque est ensuite confié sous pli scellé au commandant de bord, au chef de cabine ou à un agent de sécurité embarqué, qui remet le pli à l'arrivée à Paris-CDG à un fonctionnaire de police. La finalité de ce dispositif est de "permettre l'identification des étrangers qui, ayant présenté leurs documents de voyage et leurs visas à l'embarquement, en seraient dépourous à leur arrivée en France 63 ».

L'externalisation des contrôles qui limite les arrivées aux frontières et interdit l'accès au territoire en multipliant les «barrières à l'entrée » s'accompagne d'une privatisation grandissante des activités de contrôle, où les personnels de compagnies commerciales se retrouvent à exercer des missions de police ${ }^{64}$. Ces techniques de « dissuasion aéroportuaire » visent à ne plus laisser aucune marge aux étrangers qui seraient tentés de se «perdre» en zone dite internationale, le temps pour eux de se débarrasser de leurs papiers et de rendre impossible toute identification de leur provenance. Lors d'une visite en août 2007, nous avons rencontré un Irakien dans un local de maintien au terminal 2 qui, après une escale en Turquie, s'étonnait que les policiers soient en possession de la photocopie de son passeport alors qu'il se présentait sans papiers : c'est que l'officier de liaison à Istanbul avait bien fait son travail... Les officiers de liaison dépendent du Service technique de coopération internationale de police (STCIP), placé sous l'autorité du directeur général de la police nationale. Ces fonctionnaires, issus des directions actives de police, sont généralement placés, sur la base d'accords bilatéraux, auprès des services opérationnels du pays qui les accueille. Ils sont sous l'autorité d'un attaché de sécurité intérieure, lorsqu'il y en a un. Les officiers de liaison de la PAF accomplissent différentes tâches : diffuser les informations opérationnelles en matière de flux migratoires et de travail clandestin, se déplacer aux frontières afin de prêter assistance aux fonctionnaires qui y sont affectés, contribuer à l'harmonisation des méthodes de contrôle, etc. La collaboration active des polices de l'immigration européennes "par la mise en place graduelle d'un régime de "contrôle" communautaire 65 " constitue un facteur majeur de l'évolution des formes de confinement des étrangers en zone d'attente.

61. Article L625-3 du CESEDA.

62. Décret n²006-1243 du 11 octobre 2006, NOR : INTD0600222D.

63. Décret cité, Article 1.

64. Concernant les expulsions : L. Van Eeckhout, «Les policiers inquiets d'une privatisation du transport des étrangers retenus ", Le Monde, 4 juillet 2008.

65 . Geiger M., «Les organisations intergouvernementales et la gouvernance des flux migratoires ", Hommes et Migrations, n¹272, mars-avril 2008, p. 9. 


\section{Le maintien en « zone d'attente réservée »}

Le maintien en zone d'attente est une procédure administrative " notifiée » via un procès-verbal (une feuille de papier remise à l'étranger) sur lequel figurent la date et l'heure de la notification. Ces informations servent ensuite de référence pour le déroulement de la procédure de maintien, encadrée par un contrôle du juge judiciaire ; le maintenu est présenté au Tribunal de grande instance de Bobigny, 4 jours après son entrée effective en zone d'attente à Paris-CDG. Il est important de souligner que les étrangers confinés dans la «zone d'attente réservée » ne disposent pas toujours d'une décision de maintien ; un agent que nous interrogeons nous dit à ce propos : "je n'y suis pour rien si les supérieurs nous disent de ne pas enregistrer tous les étrangers 66 ». Ces étrangers sans décision de maintien sont confinés en transit, ne bénéficiant alors pas des droits qu'ouvre le statut de «maintenu ». Parmi ces droits, on trouve celui de "refuser d'être rapatrié avant l'expiration du délai d'un jour franc ». Depuis la loi du 2 novembre 2003, "l'étranger est invité à indiquer sur la notification s'il soubaite bénéficier $d$ u jour franc 67 ", alors qu'auparavant, l'étranger auquel était opposé un refus d'entrée ne pouvait être rapatrié contre son gré avant l'expiration de ce délai. Il n'est donc pas rare que, si le vol qui les réachemine décolle dans les quatre heures suivant leur non-admission (c'est généralement "le temps [considéré comme] strictement nécessaire à [leur] départ 68 »), les étrangers restent en aérogare sous contrôle policier et ce, sans aucune procédure. Des étrangers en transit (sauf certains en transit interrompu ${ }^{69}$ ) peuvent également être consignés de la sorte dans les postes de police et les salles de maintien des terminaux en attendant leur correspondance. "Dans le cas des étrangers "maintenus" aux frontières, il ne s'agit pas de sanctionner une infraction pénale mais de prendre une mesure de sûreté, dans le cadre d'une procédure administrative d'admission sur le territoire ${ }^{70}$. " La sûreté est la prévention et la lutte contre les actes illicites volontaires; dans le contexte d'un aéroport international de première envergure, les techniques sécuritaires de confinement des étrangers prennent le pas sur l'ancienne logique de l'enfermement disciplinaire : "ce qui compte, ce n'est pas la barrière, mais l'ordinateur qui repère la position de chacun, licite ou illicite, et opère une modulation universelle ${ }^{71}$. "

Parmi les différentes phases du parcours des étrangers en zone d'attente, nous nous intéresserons aux moments où agents de police et personnel de l'aé-

66. Compte rendu de la visite du 10 mai 2007, au T2C.

67. Article L213-2 du CESEDA.

68. Article L221-1 du CESEDA, op. cit.

69. Transit interrompu (TI) : lorsque la compagnie aérienne ou le pays de destination refuse d'embarquer ou de laisser entrer un passager, il est en TI et réacheminé vers son pays de provenance. Cela arrive souvent avec les Chinois et les Brésiliens.

70 . Julien-Laferrière F., op. cit., p. 7.

71 . Deleuze G., op. cit., p. 246. 
roport collaborent (principalement les employés des compagnies aériennes, mais aussi le personnel d'ADP et les employés des nombreux prestataires de service privés réunis sur la plate-forme aéroportuaire de Paris-CDG : sécurité, restauration, nettoyage, etc.). Depuis la transmission des données personnelles de leurs passagers à l'arrivée à Roissy jusqu'à la préparation du plan de vol qui réachemine l'étranger, le personnel d'Air France s'apparente à des «auxiliaires de police 72 ». Toutefois, ces transactions ne sont pas « sans causer quelques heurts ", nous confie un major de la PAF 73, " car ceux d'Air France sont plus intéressés par la fluidité ». Nous aborderons également les techniques de comptage et de suivi des étrangers pilotées par la police.

"Inader»

Lorsqu'un étranger est interpellé en porte d'avion ou suspecté au poste de contrôle de vouloir entrer sur le territoire avec des documents falsifiés ou ne possédant pas suffisamment d'argent sur lui, il est amené au poste de police où les agents lui prennent ses documents de voyage et d'identité. Les vérifications nécessaires sont réalisées au poste de quart par un officier de police judiciaire. Si l'étranger ne remplit pas les critères d'admission sur le territoire (visa, ressources, hébergement), il se voit notifier un procès-verbal de non-admission. Les policiers appellent cette procédure «inader» (un non-admis est un « inad d dans le jargon du IATA ${ }^{74}$ - inadmissible person). Il s'agit d'une procédure administrative, sans décision de justice. Soit le non-admis décide ensuite « de son plein gré » de « repartir le plus rapidement possible », soit il fait valoir son droit au « jour franc » - s'il en a connaissance - afin de contacter son consulat, ses proches ou un avocat. Nous rencontrons principalement en aérogares des personnes qui «vont partir d'elles-mêmes à l'instant 75 ». Depuis la loi du 26 novembre 2003, la présence physique d'un interprète n'est plus obligatoire. Ce sont alors le plus souvent des agents de police ou des compagnies aériennes qui assurent la traduction. Un agent d'escale Air France de la cellule Amérique latine, trilingue français, espagnol et portugais, nous explique qu'il lui est souvent arrivé d'assurer la traduction : «Les policiers me demandent souvent de dire aux personnes qu'il vaut mieux qu'elles repartent maintenant et libres, sinon, elles auront des problèmes avec la justice 76 ». Les menaces ou insultes sont fréquentes lors de cette phase de la procédure : les

72. "Depuis le développement du trafic aérien, les compagnies aériennes ont été soumises à des obligations semblables. Les transporteurs maritimes et aériens ont souvent refusé d'assumer ce rôle de "shérif-adjoint", principalement parce qu'ils craignaient que la participation à des activités quasi gouvernementales n'affectent leur rentabilité. Toutefois pour ne pas être jugés coupables de cupidité pure et simple, ils préfèrent considérer la régulation des déplacements comme la tâche propre de l'Etat - ce qu'elle est en réalité. ", Torpey J., " Aller et venir : le monopole étatique des “moyens légitimes de circulation” ", Cultures E Conflits, n³1-32, 1998, pp. 63-100.

73 . Compte rendu de la visite du 14 novembre 2006 au T2E.

74. IATA : International Air Traffic Association.

75 . Compte rendu de la visite du 3 juillet 2006 au T2A.

76. Entretien avec N.S., agent Air France depuis 1999, 31 juillet 2006. 
agents de la PAF ont pour consigne d'éviter tout maintien en zone d'attente «superflu ». L'objectif est de «convaincre» les personnes de repartir par le prochain vol.

"L'immigration irrégulière ne peut être maîtrisée que dans la mesure où ses manifestations sont connues de la manière la plus précise possible 77 . » La DPAF, en sa qualité de pilier de la police de l'immigration, assure la collecte et la centralisation de l'information et la production de statistiques "pour une meilleure connaissance de l'immigration irrégulière et clandestine »; elle est chargée de l'évaluation du risque migratoire irrégulier et de la définition des objectifs dans ce domaine (voir supra). Les instruments dont dispose la PAF pour recueillir, analyser, traiter et retransmettre l'information concernant les étrangers non admis ne cessent d'être affinés et modernisés. Dans chaque poste de police des aérogares de Roissy, un tableau de suivi comptabilise les étrangers en cours de procédure par catégories: GAV (garde-à-vue), NA (non admis), TI (transit interrompu), AP (asile politique), RVE (refus de visa d'escale), TA/verif (transit accompagné / vérifications). Mais ce système de comptage « manuel » est de moins en moins utilisé, les tableaux sont souvent vierges, ou comportant de vieilles informations. Les policiers inscrivent plutôt ces étrangers dans un « registre des étrangers maintenus 78 ", après avoir récupéré leurs documents de voyage 79 et constitué pour chacun d'entre eux, des dossiers facilement repérables par le numéro de MZA (maintien en zone d'attente), numéro qui les identifiera le temps de la procédure. Lors des visites à Roissy, il nous a toujours été refusé de consulter ce registre, ce qui n’est généralement pas le cas dans les autres zones d'attente.

Cette gestion pourrait rapidement changer : la multiplication des fichiers informatisés d'étrangers, tant au niveau national qu'européen (Eurodac, SIS I et II, AGDREF, etc.), et leur future «interopérabilité » constitue une ressource décisive pour les agents de la PAF. Les mesures visant à lutter contre les arrivées illégales d'étrangers sont aujourd'hui décidées dans le cadre du rapprochement des politiques d'asile et d'immigration entre les Etats membres de l'Union européenne. La centralisation du renseignement opérationnel susceptible de faire l'objet d'une exploitation judiciaire ou administrative se fait dorénavant à ce niveau. La mise en place de l'Agence aux frontières extérieures, FRONTEX, en octobre 2005, et les opérations nombreuses menées depuis sont l'aboutissement de cette coopération dans la gestion des frontières extérieures. Un récent décret ${ }^{80}$, en date du 25 juillet 2007 , autorise le traite-

77. Circulaire du 23 août 2005 relative à la mise en place de la police de l'immigration.

78. En jargon policier, ce registre est appelé le « cahier des inads ».

79. Et ils ne le leur rendront qu'une fois dans l'avion.

80 . Décret n²007-1136 du 25 juillet 2007 portant création d'un traitement automatisé de données à caractère personnel des ressortissants étrangers qui, ayant été contrôlés à l'occasion du franchissement de la frontière, ne remplissent pas les conditions d'entrée requises et modifiant la partie réglementaire du code de l'entrée et du séjour des étrangers et du droit d'asile / NO : IMID0760282D. 
ment automatisé de données à caractère personnel des "ressortissants étrangers qui, ayant été contrôlés à l'occasion du franchissement de la frontière, ne remplissent pas les conditions d'entrée requises ». Les données personnelles du non-admis (identité, documents de voyage, numérisation des empreintes des dix doigts) pourront être conservées cinq ans, des informations plus précises relatives à la procédure administrative et judiciaire de non-admission et/ou de maintien en zone d'attente pendant 32 jours.

\section{"Accompagner (ou assister)"}

Nous souhaiterions revenir ici sur la pratique du transit accompagné, ou assisté. Il s'agit de la prise en charge non réglementée de certains étrangers en transit aéroportuaire par la PAF. Elle ne relève d'aucune procédure, mais se pratique très couramment pour les personnes dont les «parcours » de voyage semblent suspects aux policiers. Informés par les agents des compagnies aériennes qui leur communiquent les trajets, les agents de la PAF interceptent directement en porte d'avion les passagers effectuant un transit par la France entre deux destinations lointaines et «peu probables ». Ils s'assurent ainsi, lorsqu'ils estiment qu'il existe un « risque migratoire » sérieux, que l'étranger n'essaiera pas de profiter du transit pour rester en France. L'objectif de la police est de lutter contre les filières d'immigration illégales et "d'éviter l'évaporation 81 . Pendant ce " transit accompagné », un agent récupère les papiers et documents de voyage de la personne « assistée » et les photocopie. Cette pratique informelle est très courante sur des vols en provenance de ou vers la Chine (une dizaine de passagers par vol sont en moyenne concernés). Là encore, les agents des compagnies aériennes sont mis au courant et facilitent le travail de la PAF. Le « délai du transit assisté ne peut être excessif » et ne doit pas excéder quatre heures ; les personnes « accompagnées » pendant leur correspondance entre un vol et l'autre attendent pourtant généralement le temps qu'il faut, et parfois une journée entière dans un local de maintien (voire plus, dans les situations exceptionnelles). Il faut noter que le transfert d'un étranger d'un lieu à l'autre de la zone d'attente, entre les différents locaux de maintien, la ZAPI 3 et les aéronefs, nécessite d'importants moyens logistiques (véhicule

81. «M. TOPIN réaffirme qu'il ne peut évoquer que la seule situation des étrangers placés en zone d'attente. Seuls ce placement et la non-admission sont des faits générateurs qui ont des conséquences au plan juridique. Les autres étrangers font l'objet de contrôles de police et il n'appartient pas aux associations d'en connaître [le document est ainsi rédigé, il manque la suite]. Mme ARAGNOUET réexplique, s'agissant du délai de 4 beures, que les étrangers concernés sont en transit à Roissy et ne demandent pas l'entrée sur le territoire. Le but du voyage est ailleurs. Il s'agit d'éviter l'évaporation. ", "Compte rendu de la réunion du 5 juillet 2006 avec les associations humanitaires sur le fonctionnement des zones d'attente, rédigé par le MIAT », p. 7, document non public. Le compte rendu 2007 de la « réunion entre administration et organisations humanitaires sur le fonctionnement des zones d'attente » est disponible sur le site de l'Anafé :

www.anafe.org/download/generalites/CR\%20r\%E9union\%20annuelle-version-assoc-1604-07.pdf 
disponible, escorte, etc.). Ces étrangers en «transit assisté » sont alors confinés sous contrôle policier, sans qu'aucune procédure administrative ne leur soit notifiée. Ils n'ont donc aucun droit. La PAF justifie ce maintien en affirmant qu'il s'agit de personnes qui demandent un visa d'escale ; ce dernier leur étant refusé, elles sont non admises en France, sans vraiment l'être, puisqu'on ne leur notifie pas un refus d'entrée ${ }^{82}$.

«Refouler 83 »

Lors de la procédure de renvoi des personnes maintenues en ZAPI 3 ou dans les aérogares, des agents de police viennent les chercher « une heure et demie » avant le départ de l'avion; elles sont conduites dans un fourgon qui les emmène directement sur les pistes de départ. Ce délai varie beaucoup en fonction des vols, des autres maintenus, des véhicules et du personnel disponibles, etc. Il n'est pas rare qu'il soit largement dépassé. La procédure d'embarquement des étrangers dépend du nombre de leurs refus d'embarquer précédents, de s'ils sont escortés ou non. Lors du refoulement des personnes non admises 84 (et des demandeurs d'asile déboutés à la frontière), la PAF est en contact avec la compagnie aérienne avec qui elle prévoit, la veille généralement, le plan de vol. Il s'agit de connaître le nombre de personnes refoulées qu'il y aura dans l'avion, et si elles seront ou non escortées par des agents de la PAF. Il est d'usage de placer la personne renvoyée au fond de l'aéronef et, si le vol n'est pas complet, de laisser une rangée libre entre cette personne et son escorte, et les autres passagers. Le langage utilisé pour ces tractations est composé des abréviations de l'IATA : les personnes refoulées sont des «DEPOs » (deported persons), qui se déclinent en "DEPUs» (unaccompanied, sans escorte) et "DEPAs » (accompanied, avec escorte) ${ }^{85}$. En effet, l'éloignement par voie aérienne connaît deux niveaux de sécurité : le départ sans escorte avec accompagnement jusqu'au pied de l'avion et le départ sous escorte jusqu'à destination. L'escorte est obligatoire pour les expulsés ; dans les autres cas, la décision d'escorte jusqu'à destination sera prise en fonction des circonstances. Une note d'instruction de la Direction générale de la police nationale relative à l'éloignement par voie aérienne 86 a été publiée suite à la mort de deux étrangers 87 en 2002 et 2003 lors de leur renvoi forcé après leur passage en zone

82. "Ils n'ont rien de spécial, mais on les accompagne pour ne pas qu'ils se perdent... », compte rendu de la visite du 2 août 2006 au 2 F.

83. Le terme de "refoulement» pose problème à certains agents de la PAF : «refouler, non... refouler, bon... oui, "refouler" mais entre guillemets. ", compte rendu de la visite du 18 mai 2007 au T1.

84. Les frais de rapatriement et d'escorte des personnes non admises sont à la charge de la compagnie aérienne ayant transporté l'étranger en France.

85. Entretien de M.J., agent chez Air France depuis 1996, le 9 août 2006.

86. Note d'instructions DGPN/DICCILEC/DIR n96-09872 du 28 novembre 1996, cité par le CPT

87. L’Argentin Ricardo Barrientos, décédé le 30 décembre 2002 et le Somalien Mariam Getu Hagos, décédé le 18 janvier 2003. 
d'attente. Elle précise que les mesures physiques de coercition utilisées lors de la mise à exécution des renvois forcés doivent être mises en œuvre avec «la force strictement nécessaire». En ce qui concerne l'acheminement à l'avion, ce mémento précise aux fonctionnaires accomplissant des missions d'escorte qu'il «convient d'adapter la force et les moyens utilisés à la dangerosité, au comportement actuel et aux antécédents de l'individu. L'emploi de la force ne signifie pas porter des coups mais maîtriser l'individu ». L'ensemble des gestes et techniques professionnels d'intervention (GTPI) ayant trait au renvoi forcé y sont consignés, parmi lesquels on trouve la technique «de contrainte et de régulation phonique » et celle de «la ceinture de contention $» 88$.

Nous ne reviendrons pas sur les nombreuses missions dont "l'exécution [ne] préserve [pas] l'intégrité de l'éloigné, des fonctionnaires et des tiers 89 ». Nous souhaiterions plutôt brièvement aborder le sujet des « tiers », en revenant sur la récente mobilisation de l'ensemble des syndicats d'Air France en faveur d'un moratoire sur les expulsions effectuées sur leurs lignes. Le 11 juillet 2007, les élus du comité central d'entreprise d'Air France, signataires d'une motion pour l'arrêt des reconduites, se sont rendus devant le siège d'Air France-KLM, appuyés dans leur démarche par de nombreuses associations, afin de convaincre direction et actionnaires de «se prononcer pour l'arrêt de l'utilisation des avions du groupe Air France - KLM pour les expulsions d'étrangers qui nuisent à l'image de la compagnie et à la sécurité des vols. » Si certains porte-parole ont insisté sur le respect des droits fondamentaux, d'autres n'ont pas hésité à mobiliser l'argument de l'entreprise socialement responsable : «C'est une manière d'alerter les actionnaires qui sont là pour gagner de l'argent sur un problème qui est aussi commercial. Les expulsions sont très mauvaises pour l'image de marque d'Air France, notamment en Afrique », a déclaré à l'AFP, Philippe Decrulle, élu CGT ${ }^{90}$. La logique commerciale aurait-elle parfois du bon ?

On se le demande vraiment à la lecture de l'article « Des expulsions fort rentables » du Canard enchaîné du 23 avril 2008, où l'on apprend que les policiers chargés d'escorter les étrangers refoulés par les airs gagnent des «miles » accordés par les compagnies aériennes à leurs clients les plus fidèles. "Sans compter que de nombreux flics bénéficient, $d u$ coup, du statut "Gold" réservé aux hommes d'affaires qui voyagent régulièrement aux frais de leur entreprise ", ajoute l'hebdomadaire. " "Je comprends que l'on soit choqué, admet une porte-parole de la Police nationale sur les ondes de RTL [... ${ }^{91}$ »; ce système d'accès aux «miles» est sur le point d'être remis en cause.

88. Voir Maugendre S., « Morts par GTPI ? », Expulser, Plein Droit, nº62, octobre 2004.

89. Note DGPN/DICCILEC/SDCCT/BE/n ${ }^{\circ} 98-05646$ du 9 juillet 1998 sur les embarquements de reconduits à bord des aéronefs, règles à respecter par les personnels d'escorte.

90. Article du Monde, 10 juillet 2007.

91 . Tiré de «Encadrer des expulsions rapporte des miles », Le Figaro, 24 avril 2008 ; voir aussi "Les policiers gagnent des points de fidélité à escorter des clandestins par avion ", Libération, 23 avril 2008. 
Le modèle d'organisation du bub de Paris-Charles de Gaulle et de son trafic en étoile a des répercussions importantes sur les techniques de confinement des étrangers utilisées dans la zone d'attente de cet aéroport. Et si notre idée est d'analyser les différentes «modulations 92 » du dispositif de la zone d'attente, entre «internat» et «contrôlat» ${ }^{93}$, nous avons vu que la zone d'attente de Paris-CDG était toujours un lieu disciplinaire d'enfermement («concentrer, répartir dans l'espace, ordonner dans le temps $\left.{ }^{94} »\right)$. Nous posons aussi l'hypothèse dans cet article que les technologies d'identification et de surveillance sécuritaires sont en passe de prendre le pas au sein du dispositif de la zone d'attente - au niveau de la gestion du contrôle à l'arrivée et du transit à Roissy tout $\mathrm{du}$ moins. L'intensification de la « circulation des personnes débouche sur des formes originales d'enfermement qui en disent long sur nos sociétés [...], qui vise[nt] moins à corriger et discipliner les individus qu'à accélérer les flux, qu'à les suivre et les anticiper 95 ». En effet, au sein du réseau aéroportuaire de ParisCharles-de-Gaulle en pleine expansion, les étrangers en " transit accompagné » font l'expérience d'un étrange service offert. Comme le dit Claude Terrazzoni, président de l'Union des Chambres de commerce et établissements gestionnaires d'aéroports (UCCEGA) lors du séminaire à l'Assemblée nationale déjà cité, «c'est que la croissance vaut bien quelques nuisances 96 ». Parmi lesquelles, le déni du droit d'asile. L'inhospitalité.

92. Deleuze G., «Post-scriptum sur les sociétés de contrôle », op. cit., p. 242 : «Les enfermements sont des moules, des moulages distincts, mais les contrôles sont une modulation, comme un moulage auto-déformant qui changerait continument, d'un instant à l'autre, ou comme un tamis dont les mailles changeraient d'un point à un autre. » (soulignement d'origine).

93 . Ibid., p. 24.

94 . Ibid., p. 240.

95 . Bigo D., «Editorial. Circuler, refouler, enfermer, éloigner : zones d'attente et centre de rétentions aux frontières des démocraties occidentales ", Cultures $\mathcal{E}$ Conflits, n³, 1996, p. 4.

96. Laurent C, op. cit. 